\section{Scanning Laser Microscope}

$W_{E}$ have designed and built a prototype of a scanning microscope for thick objects of low reflectivity and low optical contrast. As light source, we used a $5 \mathrm{~mW} \mathrm{He}-\mathrm{Ne}$ continuous wave laser (Spectra-Physics Model 120). An improved prototype is under construction; in a year or two we expect to have a microscope suitable for advancing studies of brain cells in living animals ${ }^{1-4}$.

Our innovations, inspired by previous inventions ${ }^{5,6}$ include scanning with the light beam instead of moving the object itself (as in Minsky's original microscope), and taking advantage of the characteristies of parallel light to simplify the design.

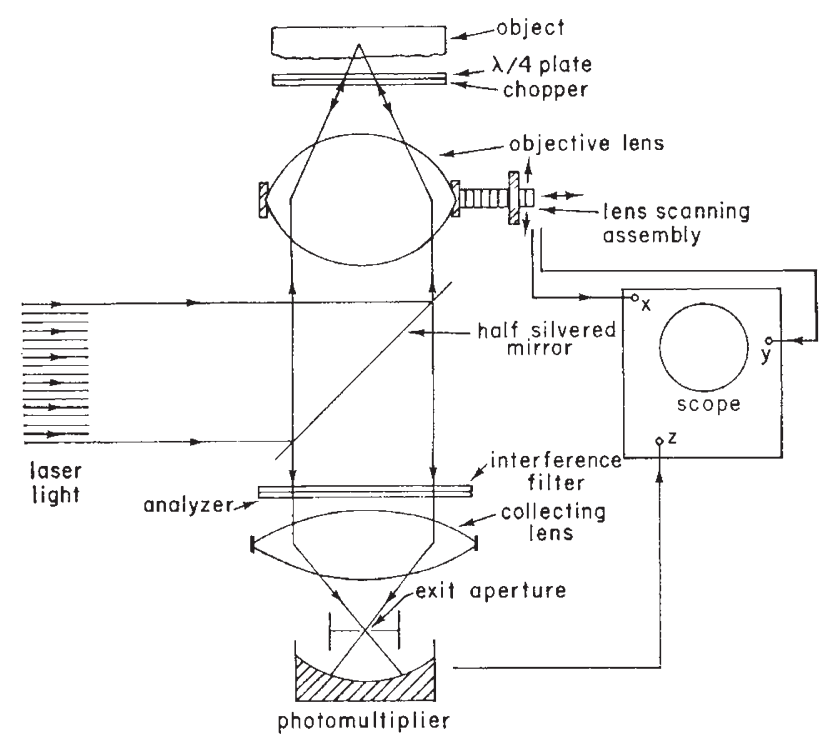

Fig. 1. Schematic of scanning laser microscope.

A schematic is shown in Fig. 1. Parallel, plane-polarized light from a laser is reflected from a half-silvered mirror through an objective lens that focuses the light at a point in the specimen, with scanning by motion of the objective lens in the desired pattern. For high magnification microscopy, the total excursion of the scan in any direction need not exceed $0.1 \mathrm{~mm}$.

The light reflected from the specimen at the principal focal point of the objective is collected by the objective and projected in a parallel beam. The reflected light is collected by a large-diameter fixed lens. Reflected light originating from the principal focal point of the scanning objective is focused at the principal focal point of the collecting lens, where there is a pinhole exit aperture transmitting light to a photomultiplier detector which controls the intensity of an electron beam scanned in synchronism with the mechanically scanned light beam. An image is built up, point by point, on an oscilloscope sereen.

Because only light reflected from the primary focal point of the objective is brought to a focus and selectively passes through the pinhole, it is possible to create a virtual "thin section" in the plane of interest within a thick specimen, screening out flare-light and scattered light that would degrade disastrously the image in a conventional microscope. Because the image is built electronically on an oscilloscope screen from electrical signals generated in a photomultiplier, we can use modern image-enhancing techniques?

To obtain an image it is absolutely necessary to suppress internal reflexions, as well as unwanted modulation effects caused by superposition of reflexions in coherent light.
We take advantage of the plane polarization of the laser's light and add a quarter-wave plate and analyser appropriately, so that reflexions internal to the microscope, and all coherent superpositions occasioned by these internal reflexions, are suppressed. The crossed analyser in front of the exit pinhole intercepts all light that has not been shifted $90^{\circ}$ by two transits through the quarter-wave plate mounted just beyond the objective ${ }^{8}$.

Further suppression of internal reflexions and their interactions is provided by a chopper and lock-in amplifier system (only the chopper is shown in Fig. 1). Only light reflected from the object will arrive at the photomultiplier alternating at the frequency of the chopper; correspondingly, only electrical signals from the photomultiplier that are alternating at the chopper frequency will be passed by the amplifier to the image display tube.

Because the image will be formed point by point from light reflected, at any instant, only from the principal focal point of the lens, coherent superpositions should not be troublesome, as they can be in attempts to form an extended image with coherent light.

Modifications of the microscope might incorporate pulsed lasers, so that very high instantaneous light energies can be used with the dissipated power below threshold for damage to delicate tissues. Pulsed ultraviolet lasers might make possible ultraviolet absorption studies in vivo ${ }^{9}$. Addition of fluorescent dyes might facilitate studies of nervous activity in vivo ${ }^{10}$.

Our microscope could also be redesigned for transmitted light microscopy, and simple modifications would make possible variations like dark field or phaso microscopy ${ }^{5}$. All reflexions along the central axis of the objective (for example, from a highly reflecting surface in front of an object) could be eliminated by mounting a small axial stop immediately behind the objective.

Research applications might include observations of other buried tissues as well as brain cells. Clinical applications might use ultraviolet light to treat tumours selectively, avoiding much destructive surgery. Diagnosis and localization of various types of tumours of the central nervous system and other organs could also be greatly facilitated; and it might be possible to make diagnostic observations of subcutaneous tissue and organs in intact organisms, without exploratory surgery or other noxious procedures.

We thank Professor W. R. Bennett, jun., Dr R. Galambos, W. Gezari, Dr M. Hadravsky and Dr M. Petrán for discussions leading to the formulation of the proposed microscope, and S. Baer for suggesting the application of a laser to Minsky's microscope. The Department of Engineering and Applied Science provided funds.

\section{Paul Davidovits}

Department of Engineering and Applied Science,

\section{David EGGer}

Department of Anatomy,

Yale University,

New Haven, Connecticut.

Received March 17; revised June 30, 1969.

'Katsuki, Y., Suga, N., Nomoto, M., and Nakatsubo, T., Proc. Japan Acad., 37, 588 (1961).

2 Egger, M. D., and Petrán, M., Science, 15\%, 305 (1967).

${ }^{3}$ Petrán, M., Hadravský, M., Egger, M. D., and Galambos, R., J. Opt. Soc. Amer., 58, 661 (1968).

${ }^{4}$ Katsuki, Y., and Kanno, Y., Jap. J. Physiol., 18, 391 (1968).

- Minsky, M., US Patent, 3,013,467, Microscopy Apparatus, Dec. 19, 1961 (filed Nov. 7,1957 ).

6 Petrán, M., and Hadravsky, M., Czech."Patent Application, 7,720 (1966).

' Nathan, R., Jet Propulsion Laboratory Tech. Rep., No. 32-877 (1966).

8 Francon, M., Progress in Microscopy, 131 (Row, Peterson, Evanston, 1961).

' Hyden, H., in The Cell (edit. by Brachet, J., and Mirsky, A. E.), 4, 215 (Academic Press, New York, 1960).

10 Tasaki, I., Carnay, I., and Sandlin, R., Science, 163, 683 (1969). 